'S Claudia, ${ }^{2} \mathrm{~B}$ Neamtu, ${ }^{2} \mathrm{H}$ Mirela, ${ }^{3} \mathrm{MBI}$ Octavia, ${ }^{4} \mathrm{M}$ Ionela, ${ }^{5} \mathrm{NM}$ Leonida, ${ }^{6} \mathrm{M}$ Melaniea, 5 Sorin. 'Research, Pediatric Hospital Sibiu; ${ }^{2}$ Research, Pediatric Hospital, Lucian Blaga University Sibiu; ${ }^{3}$ Pediatric Hospital, Lucian Blaga University of Sibiu; ${ }^{4}$ Applied Informatics, Faculty of Science, Lucian Blaga University; ${ }^{5}$ Pediatric Hospital, Lucian Blaga University; ${ }^{6}$ Laboratory Research, Pediatric Hospital, Lucian Blaga University Sibiu, Sibiu, Romania

Background Corticosteroids with or without ephedrine may have positive effects in viral pneumonia treatment accelerating the time to resolution of symtpoms but the evidence is not strong enough to make specific recommandations.

Objectives Evaluating the hospital length stay of the children with viral pneumonia treated with corticosteroids, single or in combination with ephedrine.

Material and Methods The retrospective study included pacients admitted in our clinic with viral pneumonia during 2011. From 167 cases, 78 cases were selected according to specific criteria: patients having received cortisone (hydrocortisone hemisuccinate and/or fluticasone propionate) associated or not with ephedrine (aerosol therapy) and no previous corticotherapy before admittance. Three groups have emerged: group $\mathrm{A}$, children treated with hydrocortisone hemisuccinate (27 cases), group B, children treated with hydrocortisone hemisuccinate and fluticasone propionate (27 cases), group $\mathrm{C}$, children treated with hydrocortisone hemisuccinate and aerosol therapy with ephedrine (24 cases).

A comparative analysis for the three groups regarding hospital length stay, days of treatment for each medication, $\mathrm{C}$ reactive protein values and hemogramme profiles has been made using ANOVA test.

Results The doses of corticosteroids were similar in all the groups with no statistical differences. Hospitalization period was significantly reduced $(p<0.05)$ in patients receiving hydrocortisone hemisuccinate for a longer period and significantly $(p<0.05)$ increased in children with marked lymphocytosis.

Association of ephedrine to corticosteroids didn't reduce the hospitalization period.

Conclusions Corticosteroids could be recommended for a longer period during hospitalization, for their positive effects in accelerating the time to resolution of symtpoms.

\section{AUDIT ON PRESCRIPTION OF CONTROLLED DRUG}

doi:10.1136/archdischild-2012-302724.1540

A Kage. Community Paediatrics, St Mary's Hospital, Kettering, UK

Background Prescriptions for controlled drugs are subject to prescription requirements as per UK Department of Health guidance (June 2006). A Pharmacist is not allowed to dispense a Controlled Drug unless all the information required by law is given on the presrciption.

Aim

1. To analyse prescription practice in the department

2. To analyse different formulations of Methylphenidate and Melatonin prescribed.

Methods Retrospective audit. All prescriptions between October 2011 and January 2012 analysed. Prescriptions compared against the standards given in British National Formulary (2011).

Results

Total prescriptions - 212

Controlled Drug prescriptions (methylphenidate) - 119

Melatonin prescriptions - 75

Incorrect prescriptions - $66(55.5 \%)$

Conclusions and Recommendations Most of the incorrect prescriptions were due to the form of the drug (tablets or capsules) not being specified.
Physicians made aware of the guidelines on prescribing controlled drug.

Re-Audit in 6 months.

\section{EVALUATION OF SEDATION - ANALGESIA BUY SCALE COMFORT B IN VENTILATED CHILDREN}

doi:10.1136/archdischild-2012-302724.1541

Z Addou. Anesthesie-Reanimation EHS Canastel, Oran University, Oran, Algeria

Introduction In children, the use of scale COMFORT B and a written protocol would allow the obtaining of an adequate level of sedation-analgesia, the adjustment of the dosages of midazolam and sufentanil, and finally to decrease the duration of sedation, mechanical ventilation and length of stay (LOS) in intensive care.

Materials and Methods Retrospective study over 1 year period in sédated and ventilated children, evaluated by scale COMFORT B.

Recorded parameters are: age, sex, underlying disease, dose of drugs, score of sedation COMFORT B, duration of mechanical ventilation (MV) and LOS

Results A total of 72 (27\%) ventilated children and sedated on 380 hospitalized children, 25 patients who benefited from evaluation by the scale COMFORT B according to protocol.

$66 \%$ were infants, $48 \%$ had infectious disease.

The association of drugs for sedation-analgesia were (64\%) HYPNOVEL SUFENTANIL.

The mean evaluation with scale comfort B were 6 to 8 .

The mean score of COMFORT B in the 6eme hour before protocol were; (36\%) had adequate level, (48\%) had an excessive level of sedation-analgesia, $(16 \%)$ had an insufficient level, the mean duration of ventilation was 6 days and the mean duration of (LOS) was 9 days. After protocol (94\%) had adequate level and (4\%) had inadequate level, the mean duration of (MV) was 3 days and LOS 6.8 days.

Conclusion The evaluation by the scale comfort B, would allow to adjust the dosages of midazolam and sufentanil, and to reduce the duration of ventilation and LOS

\section{FEASIBILTY OF USING HIGH FIDELITY SIMULATION EXERCISES TO EVALUATE AND ENHANCE NEONATAL RESUSCITATION SKILLS}

doi:10.1136/archdischild-2012-302724.1542

'MA Attar, ' $\mathrm{J}$ McAllister, ${ }^{1}{ }^{2} \mathrm{HM}$ Haftel. ${ }^{1}$ Department of Pediatrics and Communicable Diseases; ' 2 Department of Medical Education, University of Michigan Health System, Ann Arbor, MI, USA

Background and Aims Pediatric house officers (HO) use neonatal resuscitation (NR) skills during their rotations in the neonatal intensive care unit (NICU). To improve HOs' competence and retention of skills in NR we implemented NR practice sessions using high fidelity simulation (HFS) twice for each $\mathrm{HO}$ during their NICU rotation. This study explored the feasibility of using HFS to assess key NR skills, both at baseline and following exposure to assess improvement.

Methods We administered two standardized NR HFS sessions for each $\mathrm{HO}(\mathrm{n}=46)$ during their NICU rotation in 2010. HOs served as team leaders in during the NR scenario. We assessed total time to complete the scenario, total time to successful intubation, and the frequency of markers of NR and teamwork skills during the first and second HFS sessions.

Results We detected multiple failures in key NR and team work skills at the initial HFS sessions, such as ineffective positive pressure ventilation (PPV) $(28 \%)$, more than one attempt of intubation (30\%), incorrect decision to start chest compressions (CC) $(30 \%)$, and failure to coordinate CC and PPV (52\%), not asking for help $(59 \%)$, roles not defined (22\%). Assessment of teamwork showed a 